\title{
Load Calculation of the Most Loaded Rolling Element for a Rolling Bearing with Internal Radial Clearance
}

\section{Radoslav Tomović $(\mathbb{D}$}

Mechanical Engineering Faculty, University of Montenegro, 81000 Podgorica, Montenegro; radoslav@ucg.ac.me

Received: 9 September 2020; Accepted: 29 September 2020; Published: 3 October 2020

\begin{abstract}
This paper presents a new model for calculation of load for the most loaded rolling element in a rolling bearing with internal radial clearance. The calculation is based on a so-called load factor. By multiplying this factor by the value of the external radial load, the load transferred by the most loaded rolling element of the bearing is obtained. The values of the load factor are shown in the tables and diagrams, which makes the model very suitable for practical use. The load factors are shown for a ball bearing as well as for a roller bearing. The model considers two support positions of the inner ring on an even and odd number of rolling elements. The new model was compared with the most commonly used models up to now. The results showed greater accuracy of the studied model.
\end{abstract}

Keywords: rolling bearing; most loaded rolling element; load distribution factor; internal load distribution; bearing deflection; radial clearance

\section{Introduction}

One of the most important tasks of rolling bearings is to transfer load from the rotary elements to the supports. This takes place through a certain number of rolling elements, from the rotating ring to the stationary one. The load that rolling elements transfer, i.e., the internal load distribution, is an essential feature of rolling bearing, in particular for the determination of static and dynamic load rating, service life, the characteristics of stiffness, the accuracy of rotation, vibration characteristics, the losses due to friction, heat calculation, etc.

It is known that the calculation of the load distribution on the rolling elements is very complicated. It is nonlinear and a statically indeterminate problem, which requires that in addition to the static equilibrium equation, additional conditions have to be introduced, based on the connection between the contact stress and deformation, according to Hertz's contact theory [1]. Furthermore, in the case of rolling bearings, the internal load distribution is a time-varying value, which is dependent on the position of the rolling elements on the raceways. Time variability, static indeterminacy, and nonlinearity have meant that the calculation of the internal load distribution can only be solved numerically, through many iterations, using Newton's iteration or a similar method. However, because of its significant importance, especially for engineering practice, a large number of authors are dealing with methods to simplify the calculation of the internal load distribution of rolling bearings, and are trying to develop as simple but sufficiently accurate methods as possible.

The first papers that dealt with these problems are related to the study by Stribeck [2]. Stribeck used the Hertzian contact theory and derived an equation for calculation of the load of the most loaded rolling element for the radial ball bearing with zero clearance. The resulting solution for rolling bearing (ball or roller) with nominal clearances was later expanded by Palmgren [3]. Stribeck's expressions are still in use for the calculation of the static load rating of rolling bearing [4,5]. However, the Stribeck's expressions have one serious drawback. They do not consider the size of the internal radial clearance, but only its presence, which is described through the so-called Stribeck's number. That number is 4.37 for the ball bearing without radial clearance, and 4.08 for a roller bearing. For bearings with clearance 
greater than 0 , Stribeck's number is 5 . Later in the calculation process, Sjövall introduced the so-called Sjövall's integral [6].

The first comprehensive mathematical model for determining the load distribution for rolling bearing was developed by Jones [7]. He observed an ideal rolling bearing with an internal radial clearance and proposed a mathematical model to determine the load distribution as a nonlinear equation system. The model of Jones was later developed by Kovalev and Narodecki [8], as well as Harris [1]. Harris [1], using Sjövall's integrals, describes the size of the contact zone through a load distribution factor $(\varepsilon)$ and solves the load distribution problem by the method of trial and error. Based on the assumed distribution factor, the deflection of the bearing is determined. In the next step, equilibrium equations are solved, and the process is repeated until the given accuracy is satisfied. Harris's model was later used for different purposes and has been further developed by a large number of authors, such as Grünberg [9,10] Houpert [11], Ricci [12] Oswald [13], Rusu and Tudose [14,15], Xiao et al. [16], Fang et al. [17], etc. However, the mathematical calculation using the Harris model implies many iterations of computers, which often causes complications in concrete applications in practice.

The great practical significance comes from a paper by a research group gathered around Professor Mitrovic [18-21]. Mitrovic and associates explored the impact of structural and tribological parameters on the load distribution and the working capacity of rolling bearing. In addition, Mitrovic explored the impact of load distribution on the performance of the rolling bearing radial rigidity, the static load ratings, and the service life [22]. Mitrovic used the load distribution factor as an indicator of the load distribution nonuniformity. Mitrovic's research was later expanded by Zeljkovic and Zivkovic [23]. The analysis presented in the resulting papers [18-21] shows that among the most important factors determining the degree of load distribution non-uniformity are a radial clearance and a bearing deflection. A higher level of a bearing deflection will provide a load transfer across a larger number of rolling elements and more even load distribution. However, a higher level of the bearing deflection requires higher values of the external radial loads. In further papers [24-29], these phenomena were studied, and it was concluded that for a given bearing, the internal load distribution depends mostly on the size of the external radial load and the internal radial clearance. In a paper by Tomović [27] are shown diagrams from which it is possible to directly determine the number of active rolling elements for a given bearing type, depending on the load applied and the internal radial clearance.

As a continuation of research that has been shown previously [24-29], in this paper a new mathematical model for calculating the load of the most loaded rolling element in a bearing with an internal radial clearance is derived and presented. The model considers a radially loaded rolling bearing. Inspired by previous papers [18-23], the proposed model for the calculation uses a load factor. Multiplying this factor with the value of the external radial load, the load carried by the most loaded rolling element of a bearing was obtained. For limit values of the bearing deflection, the values of the load distribution factor were derived and presented through the appropriate tables and diagrams. Factors of load distribution were derived for the rolling ball bearing and rolling bearing with cylindrical rollers. The presented mathematical model was compared with some of the so far most widely used models. The results of this analysis are presented in this paper.

\section{Starting Assumptions}

The starting assumptions were as follows:

- A single-row, radially loaded rolling bearing, ball, or roller is being analyzed;

- The contact surfaces of the bearing are ideally made;

- The angle between the rolling elements is constant, and it can be calculated using the equation

$$
\gamma=\frac{2 \cdot \pi}{z},
$$


- The cage excludes the interplay between the rolling elements;

- The direction of the external radial load passes through the center of the bearing rings;

- All elements of the bearing are rigid, and there is no flexion. There are only contact deformations and corresponding to Hertz's theory of elasticity [1];

- The inner ring of the bearing is movable, while the outer is fixed;

- The bearing works in the isothermal conditions.

\section{Boundary Positions of Rolling Bearing}

One of the most common simplifications used in the mathematical analysis of rolling bearing is the observation of the bearing in just one position, when the center of one of the rolling elements coincides with the direction of action of the external radial load (Figure 1a). Although from the point of load distribution, this is the most unfavorable position of support, analyzing only one position is insufficient and does not provide complete information about the behavior of the bearing in operation. Namely, during working, the set of rolling elements is constantly oscillating between two boundary positions, as shown in Figure 1. This phenomenon has been detailed in previous papers $[25,28,30]$. It is clear that, due to the different position of the sets of the rolling elements, the load distribution in the two boundary positions will be different. Therefore, the value of the contact forces and contact deformations between elements of the bearing will be different in these boundary positions. Differences in the level of contact force and contact deformation will inevitably cause the differences in other running characteristics of the bearing, such as the level of deflection, stiffness, vibration characteristics, rotation accuracy, and so on.

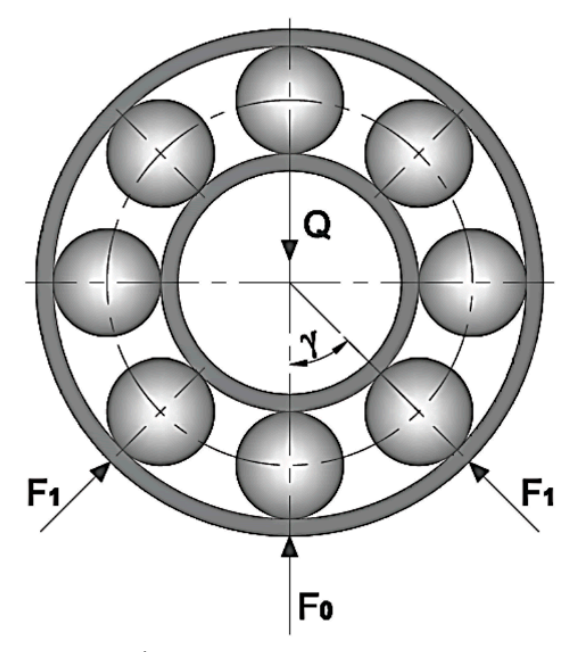

a) BSO support system

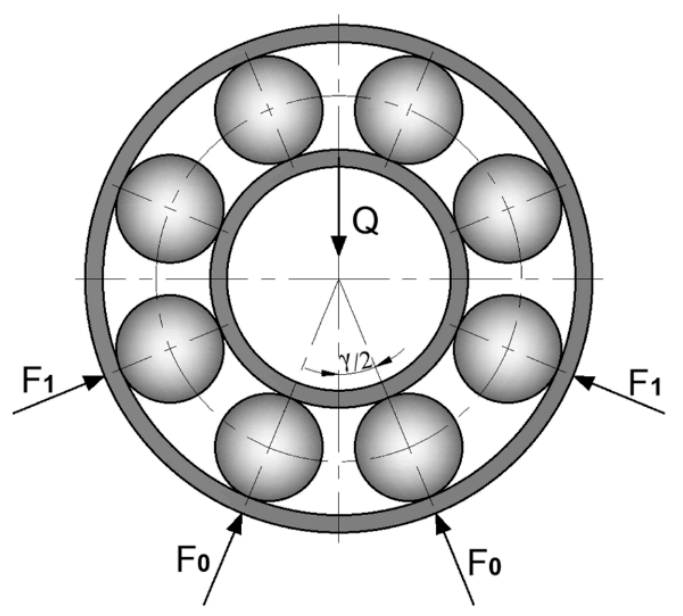

b) BSE support system

Figure 1. Boundary positions of rolling bearing.

If we want to describe as accurately as possible the behavior of the bearing in operation, then we need to introduce this second boundary support position in the analysis.

In previous studies [24-28], the following terms were adopted for these positions of support:

- The boundary position of support on an odd number of rolling elements: BSO position of support (Sl.1.a);

- The boundary position of support on an even number of rolling elements: BSE position of support (Sl.1.b).

The first boundary position of the support occurs when the center of one of the rolling elements coincides with the direction of external radial load, and the other when the rollers are symmetrically arranged relative to the direction of action of external radial load. In the following section, the value 
relating to the boundary position of support on an odd number of rolling elements will have index $o$, and the value relating to the boundary position of support on an even number rolling elements will have index $e$.

\section{Active Rolling Elements}

Active rolling elements are those elements involved in transferring the external radial load. The area bounded by the active rolling elements is called the load zone [1].

In a radially loaded rolling bearing, the load can be transferred only by rolling elements that are under the meridian plane, and only those elements that have contact with both bearing rings. This is often not the case in bearings with radial internal clearance. In bearings with radial internal clearance, due to the negative impact of clearance, it is necessary to achieve extra relative movements between the bearing rings, so that the individual rolling elements come into contact with both rings and begin to transfer external radial load. This is only possible if there is an adequate level of contact deformations between the elements of the bearing, i.e., if the bearing is loaded with the adequate intensity of external radial force.

The number of active rolling elements for a given type of bearing depends solely on the internal radial clearance and external radial loads. In [24,25], this phenomenon is explained in detail. In [26,27], the procedure for quickly and easily determining the number of active elements of the bearing is presented. The size of the necessary deflection and the necessary external radial load for entry of individual rolling elements in contact with the bearing rings can be determined on the basis of the equations shown in $[25,26]$, respectively.

Since these values are very significant for the calculation of the internal load distribution, below the basic equations for their determination will be briefly presented.

\subsection{The Maximum Number of Rolling Elements in the Load Zone of the Bearing}

The maximum number of rolling elements that can be found below the meridian plain exclusively depends on the total number of rolling elements in the bearing, and according to [25], can be calculated from the following equations:

- For the BSO position:

$$
z_{s, 0}=\frac{b-1}{2}, \quad \text { where } \quad(z-1) \leq b \geq(z+2) \&(b=3,7,11,15, \ldots)
$$

- For the BSE position:

$$
z_{s, e}=\frac{b}{2}, \quad \text { where } \quad(z-2) \leq b \geq(z+1) \&(b=4,8,12,16, \ldots)
$$

\subsection{Boundary Bearing Deflection}

Tomović [25] presented forms for the calculation of the boundary values of bearing deflection, which is required to achieve load transfer over $q$ rolling elements, as

$$
a_{q}=t_{q} \cdot \frac{e}{2}
$$

where $q$ is the number of active rolling elements of the bearing, $e$ is the radial clearance, and $t_{q}$ is the coefficient of boundary bearing deflection, obtained by the following equations [25]:

- For the BSO position:

$$
t_{q, o}=\frac{1}{\cos \frac{q-1}{2} \gamma}-1
$$


- For the BSE position:

$$
t_{q, e}=\frac{1}{\cos \frac{q-1}{2} \gamma}-\frac{1}{\cos \frac{\gamma}{2}},
$$

The coefficient $t_{q}$ can be found in tables from the previous study for reference [25].

\subsection{Boundary Load of Bearing}

The boundary external load of bearing that is required for the $q$ of the active rolling elements can be calculated as [26]

$$
Q_{q}=K \cdot s_{q} \cdot\left(\frac{e}{2}\right)^{n}
$$

where $s_{q}$ is the coefficient of the boundary bearing load, $K$ is the effective stiffness coefficient, and $n$ is the exponent ( $n=3 / 2$ for a ball bearing and $n=10 / 9$ for a bearing with the rollers). The coefficient $s_{q}$ for a BSO or BSE position can be calculated with the following equations:

$$
\begin{gathered}
s_{q, o}=\left(\frac{1}{\cos \frac{q-1}{2} \gamma}-1\right)^{n}+2 \cdot \sum_{i=1}^{\frac{q-1}{2}}\left(\frac{\cos (i \gamma)}{\cos \frac{q-1}{2} \gamma}-1\right)^{n} \cdot \cos (i \gamma), \\
s_{q, e}=2 \cdot \sum_{i=1}^{\frac{q}{2}}\left(\frac{\cos (2 i-1) \frac{\gamma}{2}}{\cos (q-1) \frac{\gamma}{2}}-1\right)^{n} \cdot \cos (2 i-1) \frac{\gamma}{2},
\end{gathered}
$$

The coefficient $s_{q}$ can be read from the tables that are shown in [26]. Boundary loads that are described by Equation (7) correspond to the bearing deflection from the Equation (4).

\subsection{Number of Active Rolling Elements}

One of the biggest problems in the calculation of load distribution in the rolling bearing is to define the number of active rolling elements $(q)$. This problem was solved using the trial and error method [1], and more iterations were required for a satisfactory solution. Using Equation (7) and the table given in a paper by Tomović [26], this number can be very easily determined. By simply comparing the applied external load to the calculated boundary values, it is possible to conclude how many rolling elements are engaged in load transfer.

\section{Load Factor of the Most Loaded Rolling Element of Bearing}

The most loaded rolling element of bearing is the one element that stands closest to the external radial force direction. In boundary positions of support are as shown in Figure 2. These are the rolling elements which carry the label zero. From the engineering point of view, the boundary support position that is shown in Figure 2a is particularly significant. In this position, the center of the most loaded rolling element lies in the direction of the external radial force. This is the most disadvantaged case for support, because the rolling elements are most loaded at the time when they find themselves on the external radial load direction, i.e., transfer the largest proportion of the external load. The maximum contact stress and deformation occur on the place of the most loaded rolling element at this position [29]. Therefore, the calculation of the largest number of bearing running performance is calculated concerning this position, such as static and dynamic load capacity, service life, etc.

In engineering practice and scientific research, only this support position is generally considered in the analyses. However, in many cases, this approach is inadequate, because it does not give enough information about the behavior of the bearing in operation. Namely, a set of rolling elements constantly changes its position during operation of the bearing, which causes a temporal change of the internal load distribution, as well as the temporal change of the intensity of contact stress and deformation and the stiffness of the bearing [31,32]. Therefore, the research presented in [24-28] proposed the 
introduction of another one boundary position, shown in Figure $2 b$. The analysis of load distribution, which is shown in this paper, will be made regarding these two boundary positions.

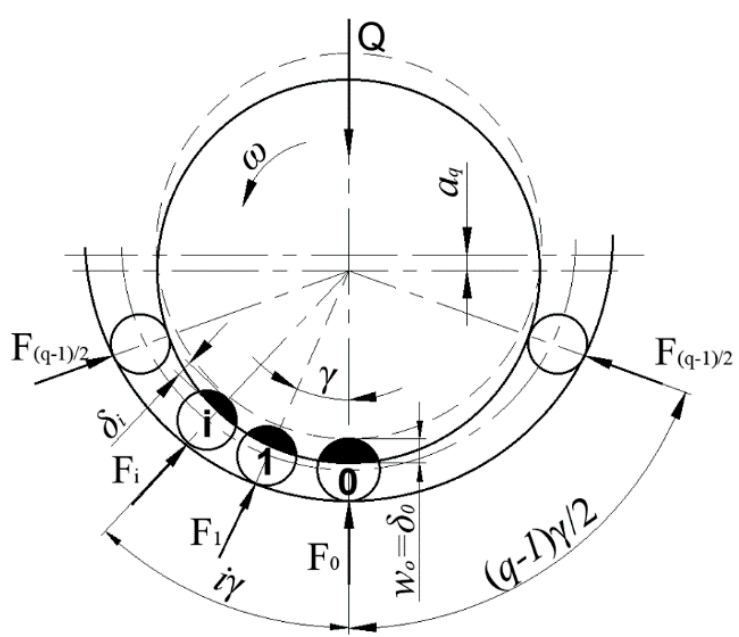

a) BSO support system

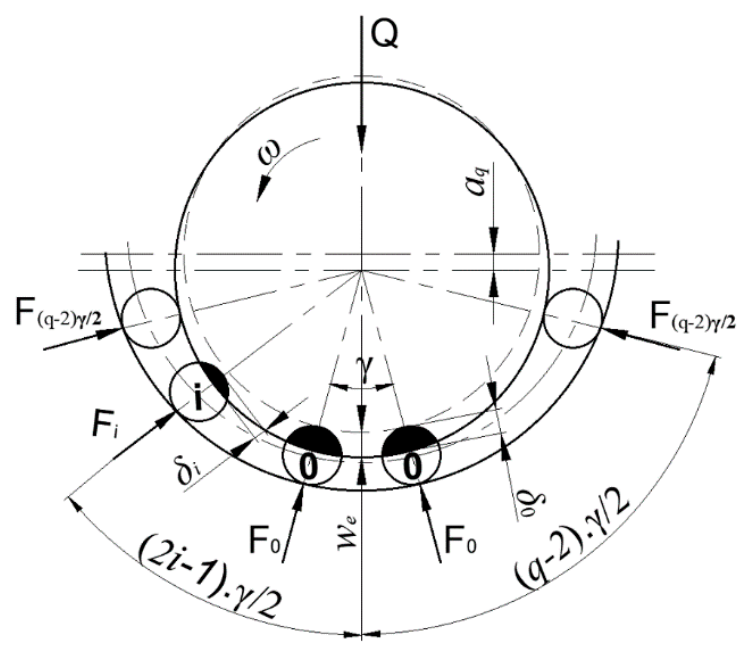

b) BSE support system

Figure 2. The load distribution for two boundary positions of bearing support.

In both of the positions shown above, the rolling elements are arranged symmetrically relative to the vertical axis, representing the direction of action of the external radial force. On this basis, it can be assumed that the load distribution is symmetrical to the external load direction and the vertical bearing axis. This creates the possibility of adopting a simple labeling system, as shown in Figure 2. The most loaded rolling element is marked with 0 . The other rolling elements have been given labels in ascending order relative to their position relative to the vertical bearing axis.

\subsection{BSO Position of Support}

The equation of equilibrium for the BSO support position can be written as (Figure 2a)

$$
Q=F_{0}+2 \cdot \sum_{i=1}^{\frac{q-1}{2}} F_{i} \cdot \cos (i \gamma),
$$

where $Q$ is the external radial load, $F_{0}$ is the load of the most loaded rolling element, $F_{i}$ is the load of the $i$-th rolling element, $\gamma$ is the angular spacing between rolling bearing elements, and $q$ is the number of active rolling elements.

The load transferred by the $i$-th rolling elements is equal to

$$
F_{i}=K \cdot \delta_{i}{ }^{n}
$$

where $\delta_{i}$ is the contact deformation at the position of the $i$-th rolling element.

Relative to Equation (11) the load of the 0 -th rolling element is equal to:

$$
F_{0}=K \cdot \delta_{0}{ }^{n}
$$

By dividing Equation (11) by Equation (12), the following can be obtained:

$$
\frac{F_{i}}{F_{0}}=\left(\frac{\delta_{i}}{\delta_{0}}\right)^{n}
$$


According to [26], the contact deformation $\delta_{i}$ is obtainable as

$$
\delta_{i}=\left(\delta_{o}-a_{2 \cdot i+1}\right) \cdot \cos (i \gamma),
$$

where $a_{2 \cdot i+1}$ is the boundary-bearing deflection until entering of $i$-th rolling element in contact with bearing rings.

Furthermore, if Equation (14) is included in Equation (13), the relationship between the load of the most loaded and the arbitrary rolling element is obtained:

$$
F_{i}=F_{0}\left[\frac{\left(\delta_{o}-a_{2 \cdot i+1}\right) \cdot \cos (i \gamma)}{\delta_{0}}\right]^{n}
$$

Since this is for the BSO support position, the contact deformation of the most loaded rolling element is equal to total deflection of the bearing, i.e., $\delta_{0}=w_{0}$; thus, Equation (15) can be written as

$$
F_{i}=F_{0}\left[\frac{\left(w_{0}-a_{2 \cdot i+1}\right) \cdot \cos (i \gamma)}{w_{0}}\right]^{n},
$$

If the above equation is inserted in Equation (10), after arranging, the relationship between the external load and the load of the most loaded rolling element was obtained, is

$$
F_{0}=\frac{Q}{1+2 \cdot \sum_{i=1}^{\frac{q-1}{2}}\left[\left(1-\frac{a_{2 i+1}}{w_{0}}\right)^{n} \cdot \cos ^{n+1}(i \gamma)\right]}
$$

If it is further taken that

$$
k_{\text {max }, o}=\frac{1}{1+2 \cdot \sum_{i=1}^{\frac{q-1}{2}}\left[\left(1-\frac{a_{2 i+1}}{w_{o}}\right)^{n} \cdot \cos ^{n+1}(i \gamma)\right]},
$$

The force which is transferred by the most loaded rolling element is obtained, as:

$$
F_{0}=k_{\max , o} \cdot Q
$$

In the equation above $k_{\max , o}$ represents a load factor of the most loaded rolling element. This factor is, in fact, a ratio of the size of the external load and the load transferred by the most loaded rolling element of bearing.

In the boundary case, when switching from one support system to the other [25], the bearing rings' relative movement is equal to the boundary deflection, which is required for supporting the inner ring on the $q$ of the rolling elements, i.e., $w_{0}=a_{q}$. Then the expression for the load factor of the most loaded rolling element is obtained as

$$
k_{\text {omaxq }}=\frac{1}{1+2 \cdot \sum_{i=1}^{\frac{q-1}{2}}\left[\left(1-\frac{a_{2 i+1}}{a_{q}}\right)^{n} \cdot \cos ^{n+1}(i \gamma)\right]},
$$

Further taking Equation (4) into account, after arranging, the above equation is obtained in its final form:

$$
k_{\text {omaxq }}=\frac{1}{1+2 \cdot \sum_{i=1}^{\frac{q-1}{2}}\left[\left(1-\frac{t_{2 i+1}}{t_{q}}\right)^{n} \cdot \cos ^{n+1}(i \gamma)\right]},
$$


Respectively, if the expressions for $t_{q}$ from Equation (5) are taken into account, then, after arranging, we can obtain

$$
k_{\text {omaxq }}=\frac{1}{1+2 \cdot \sum_{i=1}^{\frac{q-1}{2}}\left\{\left[\cos i \gamma-\frac{(1-\cos i \gamma) \cdot \cos \frac{q-1}{2} \gamma}{1-\cos \frac{q-1}{2} \gamma}\right]^{n} \cdot \cos (i \gamma)\right\}} \text {. }
$$

\subsection{BSE Position of Support}

The labeling system for the BSE position of support is shown on Figure $2 b$. The condition of force equilibrium for this boundary position can be expressed by the following equation:

$$
Q=2\left[F_{0} \cdot \cos \frac{\gamma}{2}+\sum_{i=1}^{\frac{q-2}{2}} F_{i} \cdot \cos (2 i+1) \frac{\gamma}{2}\right]
$$

Again, the most loaded rolling elements carry the 0 marks. Their contact deformations $\left(\delta_{0}\right)$, according to [19], can be calculated as follows:

$$
\delta_{0}=w_{e} \cdot \cos \frac{\gamma}{2}
$$

According to [26], contact deformation $\delta_{i}$ can be obtained as

$$
\delta_{i}=\left(w_{e}-a_{2 i+2}\right) \cdot \cos (2 i+1) \frac{\gamma}{2}=\left(\frac{\delta_{0}}{\cos \frac{\gamma}{2}}-a_{2 i+2}\right) \cdot \cos (2 i+1) \frac{\gamma}{2},
$$

If Equation (25) is now inserted into (13), the relation between the loads of the most loaded rolling element and on the arbitrary rolling element is obtained, after arranging, as

$$
F_{i}=F_{0}\left[\frac{\left(\frac{\delta_{0}}{\cos \frac{\gamma}{2}}-a_{2 i+2}\right) \cdot \cos (2 i+1) \frac{\gamma}{2}}{\delta_{0}}\right]^{n},
$$

Respectively, based on Equation (25), if $w_{e}$ is included instead of $\delta_{0}$, the following is obtained:

$$
F_{i}=F_{0}\left[\frac{\left(w_{e}-a_{2 i+2}\right) \cdot \cos (2 i+1) \frac{\gamma}{2}}{w_{e} \cdot \cos \frac{\gamma}{2}}\right]^{n},
$$

By inserting the above equation into the equation of equilibrium (Equation (23)), the relationship between the most loaded rolling element and the external radial load is obtained, after arranging:

$$
F_{0}=\frac{Q}{2 \cdot\left\{\cos \frac{\gamma}{2}+\sum_{i=1}^{\frac{q-2}{2}}\left[\left(1-\frac{a_{2 i+2}}{w_{e}}\right)^{n} \cdot \frac{\cos ^{n+1}(2 i+1) \frac{\gamma}{2}}{\cos ^{n} \frac{\gamma}{2}}\right]\right\}}=k_{\text {emax }} \cdot Q
$$

where

$$
k_{\text {emax }}=\frac{1}{2 \cdot\left\{\cos \frac{\gamma}{2}+\sum_{i=1}^{\frac{q-2}{2}}\left[\left(1-\frac{a_{2 i+2}}{w_{e}}\right)^{n} \cdot \frac{\cos ^{n+1}(2 i+1) \frac{\gamma}{2}}{\cos ^{n} \frac{\gamma}{2}}\right]\right\}}
$$


Factor $k_{\text {emax }}$ is the load factor of the most loaded rolling element. In the boundary moment, when switching from one to the other the support system [25],

$$
w_{e}=a_{q} .
$$

If this is included in Equation (29), it is obtained that the $k_{e m a x}$ factor is equal to

$$
k_{\text {emaxq }}=\frac{1}{2 \cdot\left\{\cos \frac{\gamma}{2}+\sum_{i=1}^{\frac{q-2}{2}}\left[\left(1-\frac{a_{2 i+2}}{a_{q}}\right)^{n} \cdot \frac{\cos ^{n+1}(2 i+1) \frac{\gamma}{2}}{\cos ^{n} \frac{\gamma}{2}}\right]\right\}},
$$

If Equations (4) and (6) are further taken into account, the final form of the equation for the BSE position of support is obtained, as

$$
k_{\text {emaxq }}=\frac{k_{\text {emaxq }}=\frac{1}{2 \cdot\left\{\cos \frac{\gamma}{2}+\sum_{i=1}^{\frac{q-2}{2}}\left[\left(1-\frac{t_{2 i+2}}{t_{q}}\right)^{n} \cdot \frac{\cos ^{n+1}(2 i+1) \frac{\gamma}{2}}{\cos ^{n} \frac{\gamma}{2}}\right]\right\}},}{2 \cdot\left\{\cos \frac{\gamma}{2}+\sum_{i=1}^{\frac{q-2}{2}}\left\{\left[\cos (2 i+1) \frac{\gamma}{2}-\frac{\left(\cos \frac{\gamma}{2}-\cos \frac{2 i+1}{2} \gamma\right) \cdot \cos \frac{q-1}{2} \gamma}{\cos \frac{\gamma}{2}-\cos \frac{q-1}{2} \gamma}\right]^{n} \cdot \frac{\cos (2 i+1) \frac{\gamma}{2}}{\cos \frac{\gamma}{2}}\right\}\right\}},
$$

The above equations refer to the boundary moment when the $i$-th rolling element engages with the bearing rings.

\subsection{The Load Factor of the Most Loaded Rolling Element: Tables and Diagrams}

Factors $k_{\max }$ and $k_{\operatorname{maxq}}$ show which part of the external load is transferred through the most loaded rolling element. This load is relevant when determining the static load rating of the bearing.

Factor $k_{\operatorname{maxq}}$ represents the load factor of the most loaded rolling element at the boundary moment when switching from one support system to the other [25]. At this moment, the inner ring of the bearing begins to support on $q$ the rolling elements of bearing. This factor, in fact, represents a relationship between the boundary external radial load $\left(Q_{q}\right)$ and the load transferred by the most loaded rolling element. Thus, the load that is transferred by the most loaded rolling element, in the case when the inner ring begins to support the $q$ of rolling elements, is equal to

$$
F_{\max q}=k_{\max q} \cdot Q_{q},
$$

The value $Q_{q}$ presents the minimal or the boundary external radial load, which is necessary for the support of the inner ring on the $q$ rolling elements, and can be calculated using Equations (7)-(9).

The values of factor $k_{\max q}$ for the radial ball bearing are given in Table 1, and in Table 2 for the roller bearing. The mentioned factors are obtained using Equations (22) and (33) for a bearing with a total number of rolling elements $(z)$, which varies in limits from 5 to 20. In the tables, given vertically, are the maximum possible number of active rolling elements. This number is shown separately for a case of BSO $\left(z_{s o}\right)$ and BSE $\left(z_{s e}\right)$ position of support, and was obtained by Equations (2) and (3). 
Table 1. Load factor $\left(k_{\operatorname{maxq}}\right)$ of the most loaded rolling element for the radial ball bearing.

\begin{tabular}{|c|c|c|c|c|c|c|c|c|c|c|}
\hline \multirow{2}{*}{$z$} & \multirow{2}{*}{$z_{s n}$} & \multirow{2}{*}{$z_{s p}$} & \multicolumn{8}{|c|}{ The Number of Active Rolling Elements $(q)$} \\
\hline & & & 3 & 4 & 5 & 6 & 7 & 8 & 9 & 10 \\
\hline 3 & 1 & 2 & & & & & & & & \\
\hline 4 & 1 & 2 & & & & & & & & \\
\hline 5 & 3 & 2 & 1.0000 & & & & & & & \\
\hline 6 & 3 & 2 & 1.0000 & & & & & & & \\
\hline 7 & 3 & 4 & 1.0000 & 0.5550 & & & & & & \\
\hline 8 & 3 & 4 & 1.0000 & 0.5412 & & & & & & \\
\hline 9 & 5 & 4 & 1.0000 & 0.5321 & 0.5182 & & & & & \\
\hline 10 & 5 & 4 & 1.0000 & 0.5257 & 0.5010 & & & & & \\
\hline 11 & 5 & 6 & 1.0000 & 0.5211 & 0.4887 & 0.3892 & & & & \\
\hline 12 & 5 & 6 & 1.0000 & 0.5176 & 0.4796 & 0.3780 & & & & \\
\hline 13 & 7 & 6 & 1.0000 & 0.5150 & 0.4727 & 0.3694 & 0.3510 & & & \\
\hline 14 & 7 & 6 & 1.0000 & 0.5129 & 0.4673 & 0.3628 & 0.3412 & & & \\
\hline 15 & 7 & 8 & 1.0000 & 0.5112 & 0.4630 & 0.3575 & 0.3335 & 0.2913 & & \\
\hline 16 & 7 & 8 & 1.0000 & 0.5098 & 0.4595 & 0.3533 & 0.3273 & 0.2842 & & \\
\hline 17 & 9 & 8 & 1.0000 & 0.5087 & 0.4566 & 0.3498 & 0.3222 & 0.2783 & 0.2655 & \\
\hline 18 & 9 & 8 & 1.0000 & 0.5077 & 0.4542 & 0.3469 & 0.3180 & 0.2735 & 0.2594 & \\
\hline 19 & 9 & 10 & 1.0000 & 0.5069 & 0.4522 & 0.3444 & 0.3145 & 0.2695 & 0.2542 & 0.2314 \\
\hline 20 & 9 & 10 & 1.0000 & 0.5062 & 0.4505 & 0.3424 & 0.3115 & 0.2661 & 0.2499 & 0.2266 \\
\hline
\end{tabular}

Table 2. Load factor $\left(k_{\max q}\right)$ of the most loaded rolling element for the roller bearing.

\begin{tabular}{|c|c|c|c|c|c|c|c|c|c|c|}
\hline \multirow{2}{*}{$z$} & \multirow{2}{*}{$z_{s n}$} & \multirow{2}{*}{$z_{s p}$} & \multicolumn{8}{|c|}{ The Number of Active Rolling Elements ( $q$ ) } \\
\hline & & & 3 & 4 & 5 & 6 & 7 & 8 & 9 & 10 \\
\hline 3 & 1 & 2 & & & & & & & & \\
\hline 4 & 1 & 2 & & & & & & & & \\
\hline 5 & 3 & 2 & 1.0000 & & & & & & & \\
\hline 6 & 3 & 2 & 1.0000 & & & & & & & \\
\hline 7 & 3 & 4 & 1.0000 & 0.5550 & & & & & & \\
\hline 8 & 3 & 4 & 1.0000 & 0.5412 & & & & & & \\
\hline 9 & 5 & 4 & 1.0000 & 0.5321 & 0.4858 & & & & & \\
\hline 10 & 5 & 4 & 1.0000 & 0.5257 & 0.4696 & & & & & \\
\hline 11 & 5 & 6 & 1.0000 & 0.5211 & 0.4580 & 0.3705 & & & & \\
\hline 12 & 5 & 6 & 1.0000 & 0.5176 & 0.4495 & 0.3592 & & & & \\
\hline 13 & 7 & 6 & 1.0000 & 0.5150 & 0.4430 & 0.3506 & 0.3276 & & & \\
\hline 14 & 7 & 6 & 1.0000 & 0.5129 & 0.4379 & 0.3440 & 0.3178 & & & \\
\hline 15 & 7 & 8 & 1.0000 & 0.5112 & 0.4338 & 0.3387 & 0.3102 & 0.2744 & & \\
\hline 16 & 7 & 8 & 1.0000 & 0.5098 & 0.4306 & 0.3345 & 0.3041 & 0.2670 & & \\
\hline 17 & 9 & 8 & 1.0000 & 0.5087 & 0.4279 & 0.3310 & 0.2991 & 0.2611 & 0.2476 & \\
\hline 18 & 9 & 8 & 1.0000 & 0.5077 & 0.4256 & 0.3281 & 0.2950 & 0.2562 & 0.2414 & \\
\hline 19 & 9 & 10 & 1.0000 & 0.5069 & 0.4237 & 0.3257 & 0.2916 & 0.2522 & 0.2363 & 0.2171 \\
\hline 20 & 9 & 10 & 1.0000 & 0.5062 & 0.4221 & 0.3236 & 0.2887 & 0.2488 & 0.2320 & 0.2122 \\
\hline
\end{tabular}

In addition, the dependence of the coefficient $k_{\text {maxq }}$ on the total number of rolling elements in a bearing is shown in Figures 3 and 4. The different curves in the diagrams are given in relation to the different number of active rolling elements. The diagrams shown correspond to the boundary values of the bearing deflection, at which the $q$-th rolling element engages into contact with both rings, i.e., when the end two rolling elements from the zone of load begin to engage in the transfer of the external load. At that moment, these two elements have a 0 -th contact deformation, i.e., the 0 -th load. 


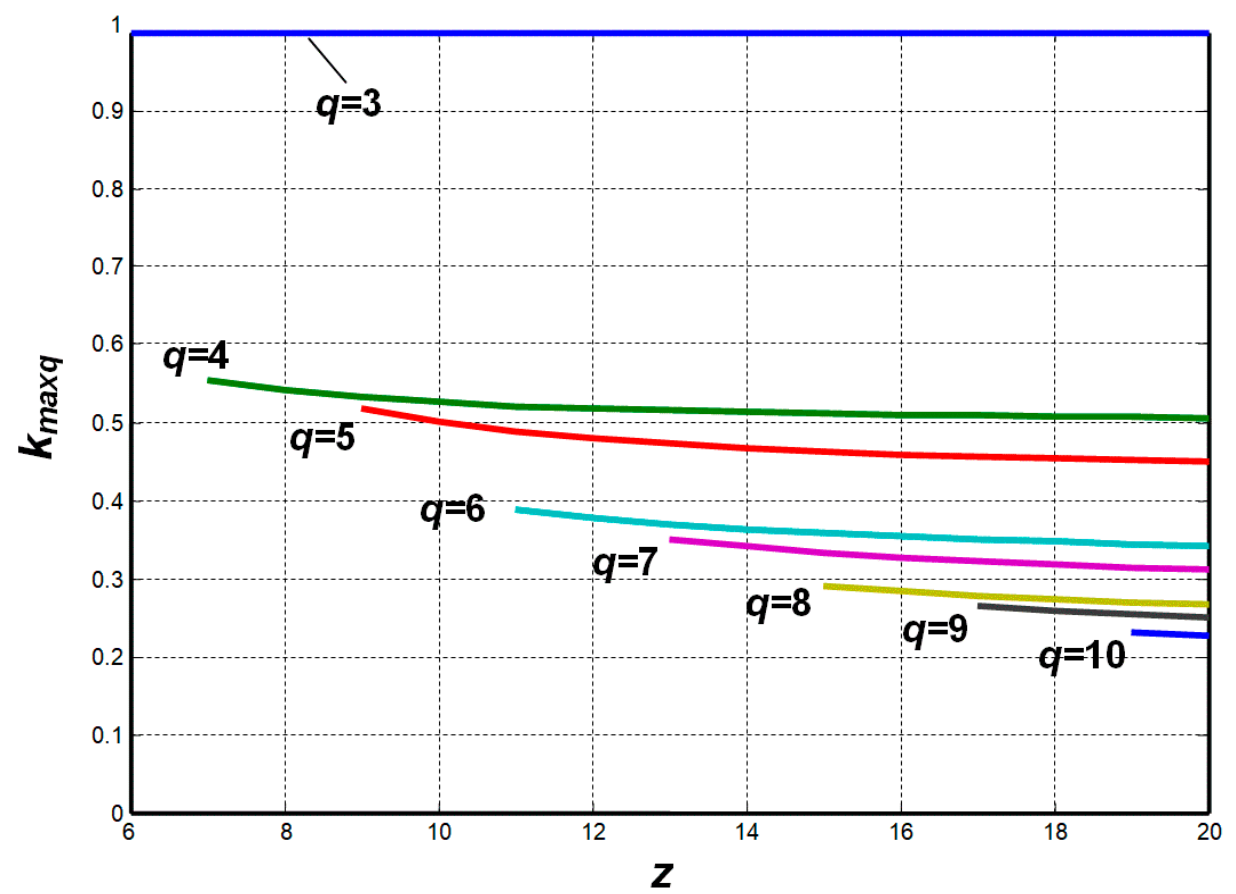

Figure 3. Load factor $\left(k_{\max }\right)$ of the most loaded rolling element for the radial ball bearing.

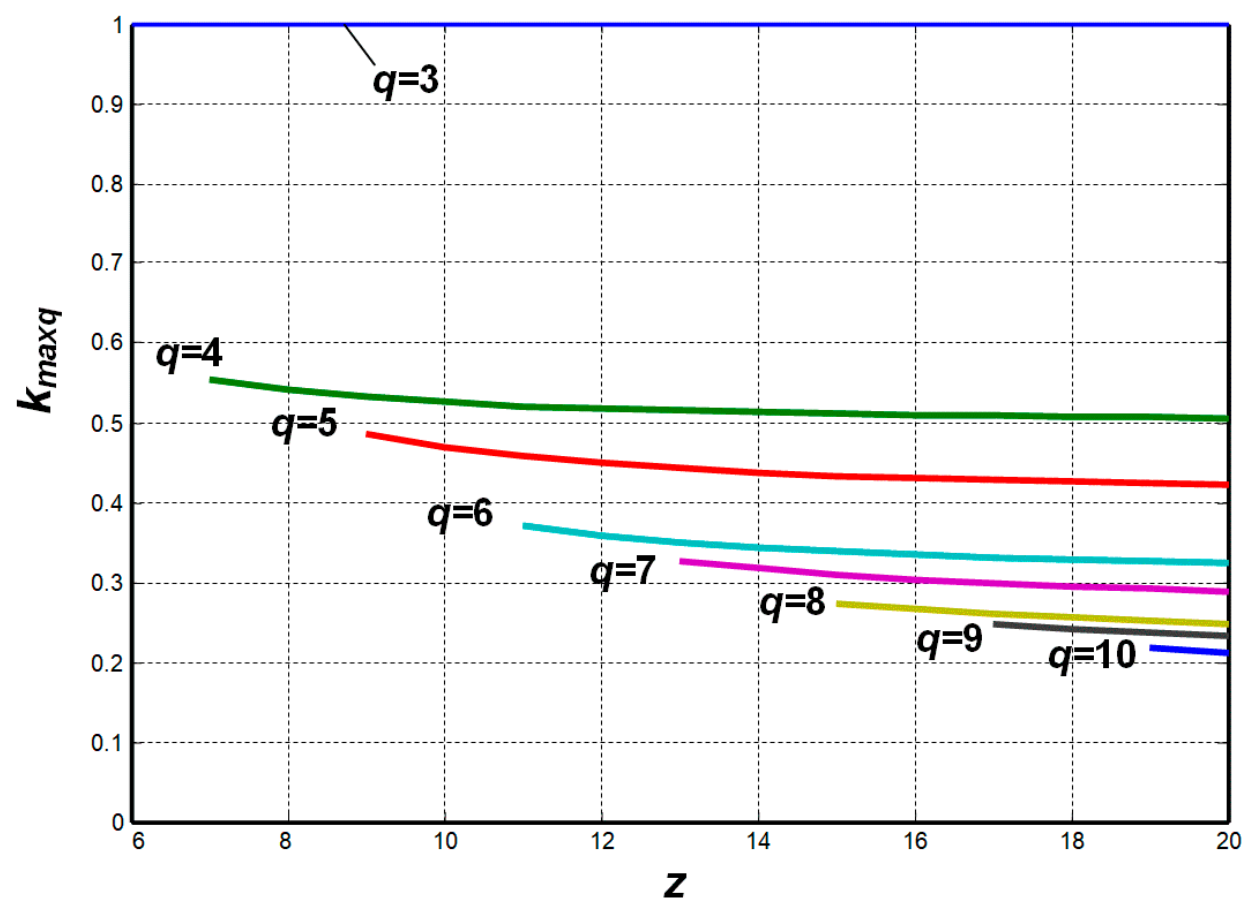

Figure 4. Load factor $\left(k_{\operatorname{maxq}}\right)$ of the most loaded rolling element for the roller bearing.

\subsection{Discussion of Equations, Tables, and Diagrams}

From Equations (22) and (33), it can be seen that factor $k_{\operatorname{maxq}}$ solely depends on the total number and number of active rolling elements. At first glance, one gets the impression that factor $k_{\max }$ is not dependent on the internal radial clearance. However, for a given external load, the number of active rolling elements is directly dependent on the internal radial clearance [27], and through this parameter, the internal radial clearance directly influences the size of the factor $k_{\max q}$.

Figures 3 and 4 clearly show that with the increase of the total number of rolling elements, the value of $k_{\max q}$ factor declines. However, this decline is inconsiderable, and it is getting smaller as 
the total number of rolling elements in the bearing increases. This means that the total number of rolling elements does not have a significant impact on the value of factor $k_{\text {maxq }}$. With regard to the value of factor $k_{\operatorname{maxq}}$, the biggest impact is the number of active rolling elements. Besides that, the image in Figures 3 and 4 show that with the increase of the total number of rolling elements in the bearing, the value of the load factor asymptotically leans toward a constant value. These statements offer the possibility of approximation and wide-open opportunities for further research and the adoption of general values for the $k_{\max q}$ factor.

From Figures 3 and 4, it can also be noted that there is a great similarity between the diagrams that show the value of factors $k_{\max }$ for the ball and for the bearing with rollers. However, the values of the factor $k_{\max q}$ at the bearings with rollers are slightly lower when supporting on $q=5-10$ rolling elements. This difference in the size of $k_{\max }$ factor occurs due to the different values of the exponent $n$, which in the ball bearing is $n=3 / 2$, and in the roller bearing is $n=10 / 9$.

\section{The Validity Analysis of the Proposed Model}

In order to verify the proposed model, a calculation of the load that transfers the most loaded roller element for ball bearing 6008 was made. The calculation was carried out in relation to the following equation:

$$
F_{0}=k_{0} \cdot Q,
$$

Here is $k_{0}$, the load factor of the 0 -th rolling element. The values of the coefficient $k_{0}$ were adopted as follows:

- In cases where the assumed external load was less than the boundary load, i.e., when $Q \leq Q_{3}$, the load transfer inside the bearing occurs via one or two rolling elements, i.e., as per support system $1[25,30]$. In these cases, for the load calculation is used the coefficient $k_{0}=k_{\max 3}=1$. By increasing the load above $Q_{3}$, new rolling elements engage in contact with the bearing rings, and the part of the load that transfers the most loaded rolling element decreases.

- For the loads which are in the interval $Q_{q}<Q \leq Q_{q+1}$, the value of the load factor is obtained by interpolation, using the next equation:

$$
k_{0}=k_{0 q}+\left(k_{0 q+1}-k_{0 q}\right) \cdot \frac{Q-Q_{q}}{Q_{q+1}-Q_{q}}
$$

The load factor values selected in this way give the most accurate calculation results.

- For the loads that are above the boundary load, corresponding to the maximum possible number of the active rolling elements, the value of the load factor $k_{0}=k_{0 z s}$ is taken, and respectively for the bearing $6008 k_{0}=k_{06}$.

The basic parameters of the ball bearing 6008, which are necessary for the calculation, are shown in Table 3. The coefficients $t_{q}$ and $s_{q}$ are taken from tables shown in $[25,26]$. The values of the load factor of the most loaded rolling element $\left(k_{0 q}=k_{\max q}\right)$ are taken from Table 1.

Table 3. The basic parameters of the ball bearing 6008 .

\begin{tabular}{ccccc}
\hline & $\boldsymbol{q}=\mathbf{3}$ & $\boldsymbol{q}=\mathbf{4}$ & $\boldsymbol{q}=\mathbf{5}$ & $\boldsymbol{q}=\mathbf{5}$ \\
\hline$t_{\mathrm{q}}$ & 0.1547 & 0.3789 & 1.0000 & 2.8284 \\
$s_{\mathrm{q}}$ & 0.0608 & 0.4278 & 2.0849 & 11.9475 \\
$k_{0 q}$ & 1.0000 & 0.5176 & 0.4796 & 0.3780 \\
\hline $\begin{array}{l}\text { The total number of rolling elements: } \\
\text { Maximum possible number of active rolling elements: }\end{array}$ & $\begin{array}{l}z_{s}=6 \\
\text { The effective coefficient of the bearing: }\end{array}$ \\
$\begin{array}{l}\text { The } \\
\text { The }\end{array}$ & $K=2.88 \times 10^{5} \mathrm{~N} / \mathrm{mm}^{3 / 2}$ \\
\hline
\end{tabular}


In Table 4 are shown the boundary values of external radial load $Q_{q}$, corresponding to values of the internal radial clearance, which are considered in this analysis and which range from 0 to $50 \mu \mathrm{m}$. In this way, the entire range of recommended internal clearance values for bearing 6008 is covered, for all classes (C2 to C5) [33]. The values of the boundary loads $Q_{q}$ were obtained using Equation (7).

Table 4. The boundary values of external radial load $Q_{q}$ for the ball bearing 6008.

\begin{tabular}{ccccccccc}
\hline$Q$ & \multicolumn{7}{c}{ The Internal Radial Clearance } \\
\hline $\mathbf{( N )}$ & $\mathbf{0}$ & $\mathbf{5}$ & $\mathbf{1 0}$ & $\mathbf{1 5}$ & $\mathbf{2 0}$ & $\mathbf{3 0}$ & $\mathbf{4 0}$ & $\mathbf{5 0}$ \\
\hline$Q_{3}$ & 0 & 2.19 & 6.18 & 11.36 & 17.49 & 32.13 & 49.46 & 69.13 \\
$Q_{4}$ & 0 & 15.38 & 43.50 & 79.92 & 123.05 & 226.05 & 348.03 & 486.39 \\
$Q_{5}$ & 0 & 74.96 & 212.02 & 389.50 & 599.67 & 1101.67 & 1696.14 & 2370.42 \\
$Q_{6}$ & 0 & 429.55 & 1214.96 & 2232.03 & 3436.43 & 6313.12 & 9719.69 & $13,583.68$ \\
\hline
\end{tabular}

The calculation results are shown in Table S1 (Supplementary Materials). Results are shown in relation to the proposed model (index $F_{0}$ ), Stribeck's method $(S)$, and by solving the system of equilibrium equations inside the bearing ( $E R$ : exact result). The equilibrium equations were derived based on the procedure shown in [25]. The solutions obtained in this way were considered accurate. In relation to these results, the error of the proposed method and the Stribeck method is obtained in the following procedure. The calculation errors, expressed as a percentage, are shown below the calculation results.

The load varied in the range from 0 to $10,000 \mathrm{~N}$, which is close to the static load rating of the ball bearing 6008 , which amounts $\mathrm{C}_{0}=11,600 \mathrm{~N}$. For the boundary loads shown in Table 4, the calculation was also made. These results are also shown in Table S1.

For efficient analysis, the results shown in Table S1 are shown in Figures 5-12. The left side of the figure shows loads which transfer the 0 -th roller bearing element. In order to compare the calculation results, the calculation error according to the proposed method and the Stribeck method is shown on the right side.
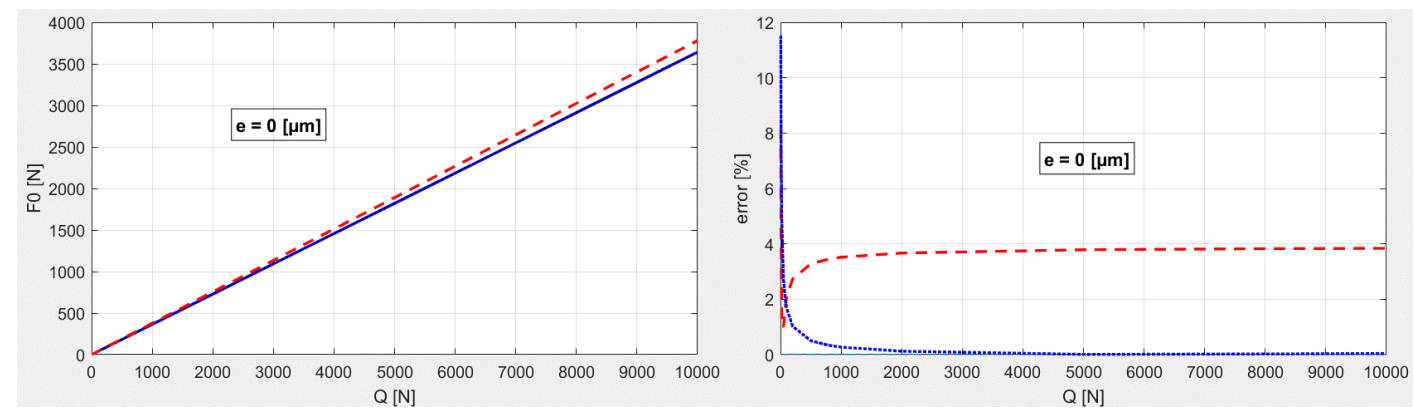

Figure 5. The load of the most loaded rolling element for ball bearing 6008; clearance $e=0 \mu \mathrm{m}$, (solid line: the correct result, dashed line: proposed method, dotted line: Stribeck's method). 

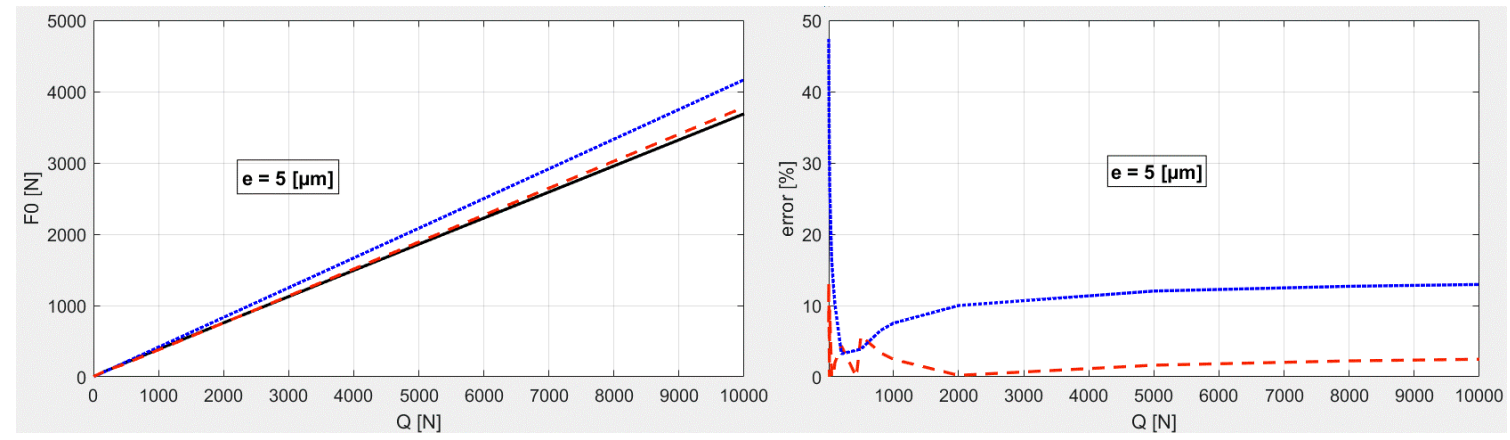

Figure 6. The load of the most loaded rolling element for ball bearing 6008; clearance $e=5 \mu \mathrm{m}$, (solid line: the correct result, dashed line: proposed method, dotted line: Stribeck's method).
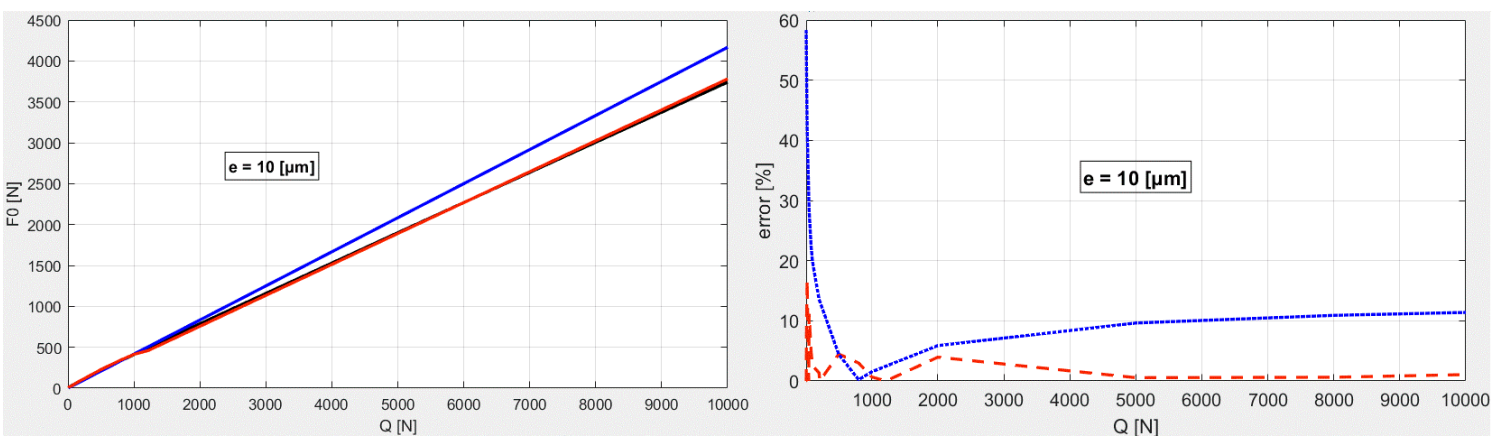

Figure 7. The load of the most loaded rolling element for ball bearing 6008; clearance $e=10 \mu \mathrm{m}$, (solid line: the correct result, dashed line: proposed method, dotted line: Stribeck's method).
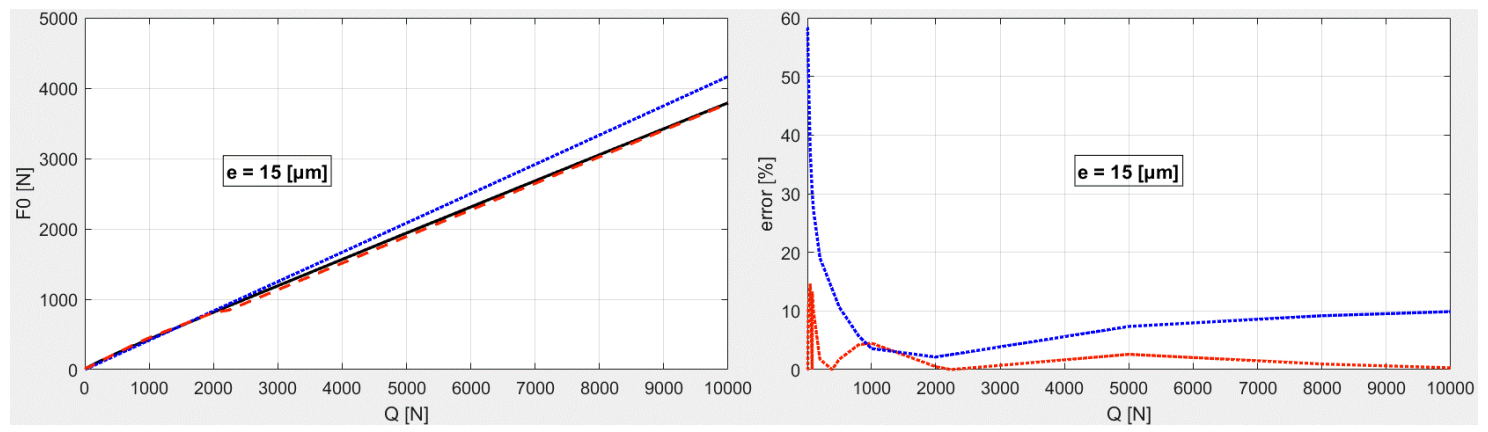

Figure 8. The load of the most loaded rolling element for ball bearing 6008; clearance $e=15 \mu \mathrm{m}$, (solid line: the correct result, dashed line: proposed method, dotted line: Stribeck's method).
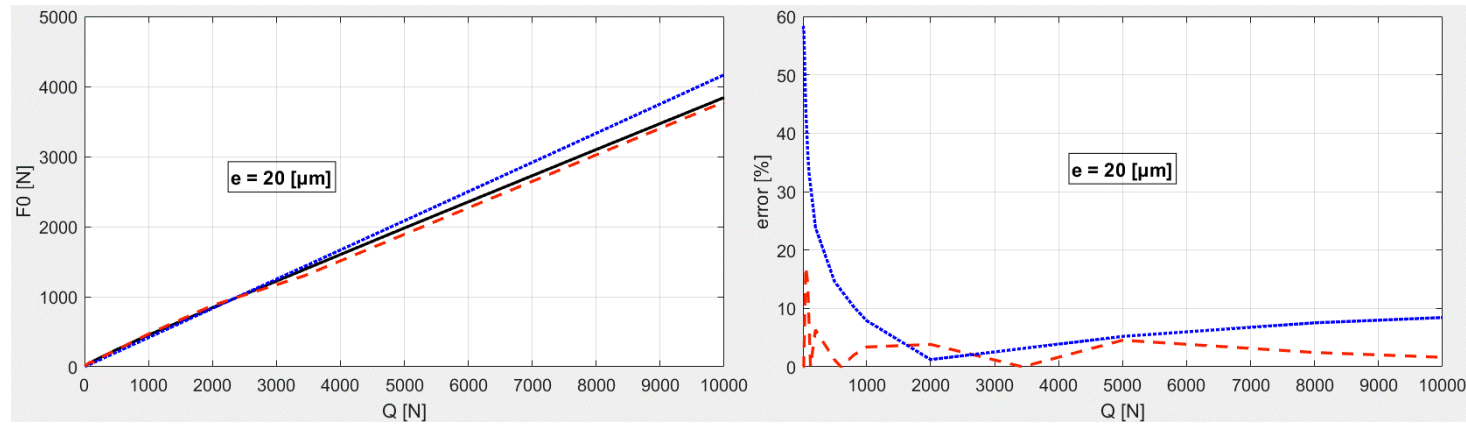

Figure 9. The load of the most loaded rolling element for ball bearing 6008; clearance $e=20 \mu \mathrm{m}$, (solid line: the correct result, dashed line: proposed method, dotted line: Stribeck's method). 

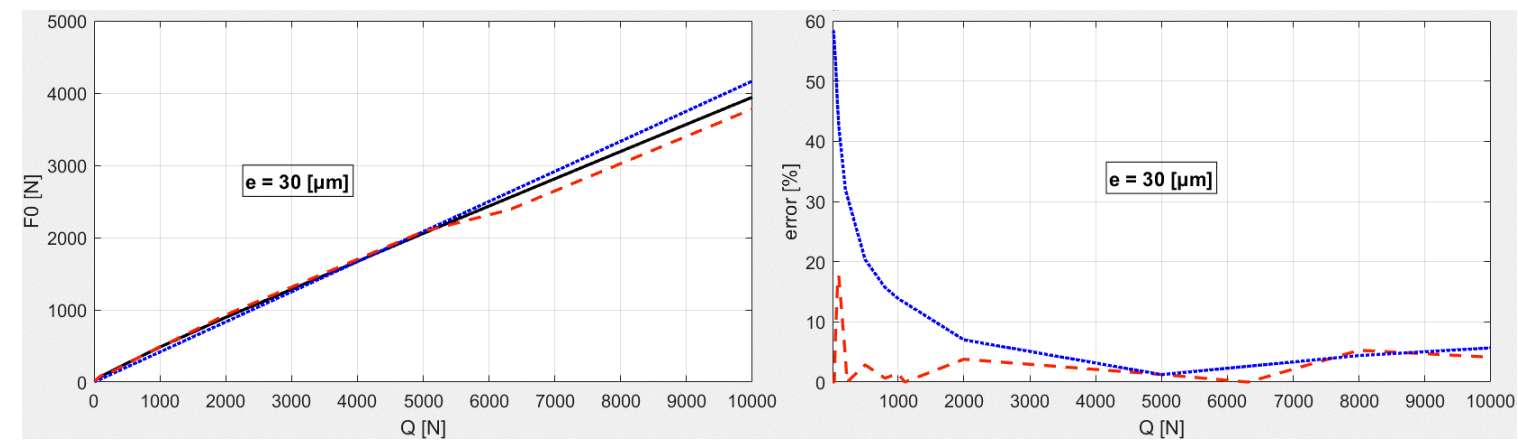

Figure 10. The load of the most loaded rolling element for ball bearing 6008; clearance $e=30 \mu \mathrm{m}$, (solid line: the correct result, dashed line: proposed method, dotted line: Stribeck's method).
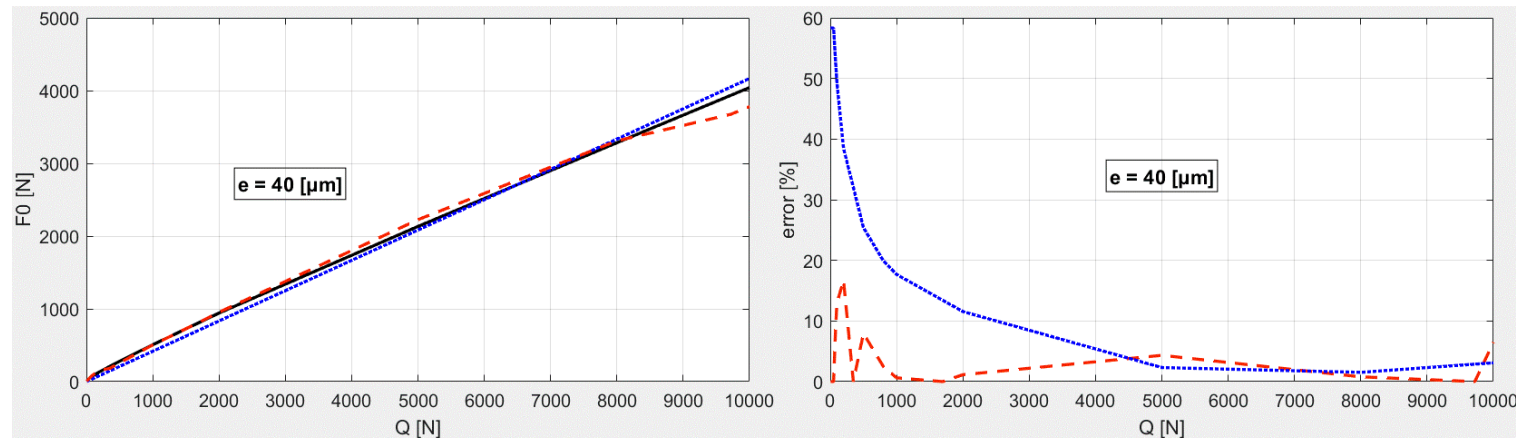

Figure 11. The load of the most loaded rolling element for ball bearing 6008; clearance $e=40 \mu \mathrm{m}$, (solid line: the correct result, dashed line: proposed method, dotted line: Stribeck's method).
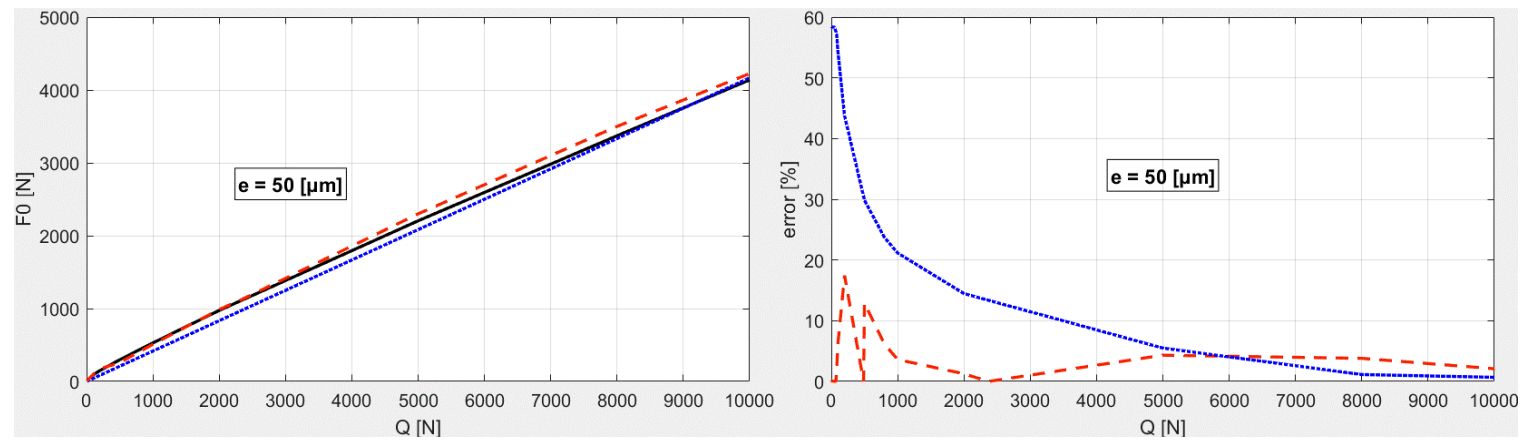

Figure 12. The load of the most loaded rolling element for ball bearing 6008; clearance $e=50 \mu \mathrm{m}$, (solid line: the correct result, dashed line: proposed method, dotted line: Stribeck's method).

\section{Conclusions}

This paper presents a new mathematical model for calculating the load transferred by the most loaded rolling element of the bearing. The model is based on so-called load distribution factors. The load factors show which part of the external radial load transfers the most loaded rolling element of the bearing.

The model is characterized by great simplicity, which makes it very suitable for practical use. By simply multiplying the load factor by the size of the external radial load, the size of the load transferred over the most loaded rolling element is obtained.

The load factors are shown in the form of tables, in particular for ball bearings and in particular, for a roller bearing, and correspond to all types of a radial loaded ball and roller bearings.

The factors in the tables can be easily read based on only two parameters: the total number of rolling elements in the bearing and the number of active rolling elements. 
The total number of rolling elements in the bearing does not have a significant impact on the size of the load factors. With regard to the size of the load factor, the most significant influence is of the number of active rolling elements. This fact creates a presumption for the adoption of general load factor values for different types of bearings. General factors for all types of bearings would only be dependent on the number of active rolling elements in the bearing.

Because of its simplicity, the calculation proposed in this paper can be compared to a calculation based on Stribeck's numbers. Both calculations are based on simple mathematical operations, do not require the usage of computers, and they are very useful for the fast calculations and fieldwork.

The analysis showed that for bearings with a radial clearance greater than zero, the results obtained by the proposed method have significantly higher precision. For bearings with a radial clearance equal to zero, the Stribeck method has greater precision.

Stribeck's number for ball bearings with zero clearance is $S=4.37$, and for bearings with internal clearance other than zero $S=5$. These values of Stribeck's numbers have been in use for many years, and represent the generally adopted value for calculating the load of the most loaded ball. For bearings with zero radial clearance, the use of a Stribeck number is fully justified. However, the analysis made in this paper indicates the need for the correction of the Stribeck's number for bearings with a radial clearance greater than zero. Specifically, Stribeck's calculation only differentiates whether or not the clearance is present in the bearing, regardless of its size. The Stribeck's number for all bearings with a clearance is the same, regardless of the size of the clearance.

The model proposed in this paper considers the impact of the clearance size on the load distribution within the bearing, through the number of active rolling elements of the bearing. In this regard, it is suggested that a correction of the Stribeck's number be made concerning the number of active rolling elements in the bearing.

Supplementary Materials: The following are available online at http://www.mdpi.com/2076-3417/10/19/6934/s1, Table S1: The calculation results of the load of the most loaded rolling element.

Funding: This research received no external funding.

Conflicts of Interest: The authors declare no conflict of interest.

\section{Nomenclature}

angle between rolling elements the maximum number of rolling elements that can be found in the loaded zone number of active rolling elements internal radial clearance deflection of bearing coefficient of boundary deflection of bearing bearing radial load boundary load of bearing Hertz's stiffness coefficient coefficient of boundary radial load rolling element load contact deformation exponent bearing deflection load factor of the rolling element load factor of the rolling element in the boundary moment load factor of the most loaded rolling element load of the most loaded rolling element 


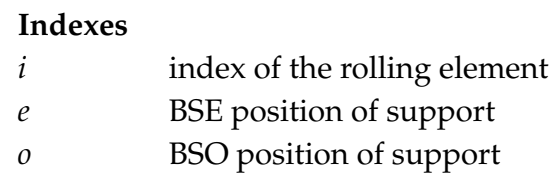

\section{References}

1. Harris, T.A.; Kotzalas, M.N. Rolling Bearing Analysis; Taylor \& Francis Group: Abingdon, UK, 2007.

2. Stribeck, R. Ball Bearings for Various Loads. Trans. ASME 1907, 29, 420-463.

3. Palmgren, A. Ball and Roller Bearing Engineering, 3rd ed.; SKF Industries: Gothenburg, Sweden, 1959.

4. International Standard ISO-76:1987. Rolling Bearings-Static Load Ratings; International Standards Organizations: Geneva, Switzerland, 1987.

5. International Standard ISO-76:2006. Rolling Bearings-Static Load Ratings; International Standards Organizations: Geneva, Switzerland, 2006.

6. The Load Distribution within Ball and Roller Bearings under Given External Radial and Axial Load. Available online: https:/ci.nii.ac.jp/naid/10027763756/ (accessed on 1 October 2020).

7. Jones, A.B. A General Theory for Elastically Constrained Ball and Roller Bearings under Arbitrary Load and Speed Conditions. ASME J. Basic Eng. 1960, 82, 309-320. [CrossRef]

8. Kovalev, M.P.; Narodecki, M.Z. Calculation of High-Precision Ball Bearings; Mahsinostroenie: Moscow, Russia, 1975.

9. Grünberg, U. Studies of the interaction between the outer ring and housing on the carrying capacity radially loaded cylindrical rolling bearings, Part 1: Theoretical basis and calculation. Construction 1970, 22, 48-55.

10. Grünberg, U. Studies of the interaction between the outer ring and housing on the carrying capacity radially loaded cylindrical rolling bearings, Part 2: Practical application and results. Construction 1970, 22, 97-103.

11. Houpert, L. A Uniform Analytical Approach for Ball and Roller Bearing Calculation. Proceedings at the STLE/ASME Tribology Conference San Francisco. ASME J. Trib. 1997, 119, 851-857. [CrossRef]

12. Ricci, M.C. Internal loading distribution in statically loaded ball bearings subjected to a combined radial and thrust load, including the effects of temperature and fit. In Proceedings of the World Academy of Science, Engineering and Technology (WCSET 2009), Amsterdam, The Netherlands, 23-25 September 2009; Volume 55, pp. 245-265.

13. Oswald, F.B.; Zaretsky, E.V.; Poplawski, J.V. Effect of Internal Clearance on Load Distribution and Life of Radially Loaded Ball and Roller Bearings. Tribol. Trans. 2012, 55, 245-265. [CrossRef]

14. Rusu, F.; Tudose, C.; Tudose, L. Influence of rotation angle on bearing rolling bodies load distribution. Part 1: Mathematical model. Acta Tech. Napoc. Ser. Appl. Math. Mech. 2013, 56, 469-474.

15. Rusu, F.; Tudose, C.; Tudose, L. Influence of rotation angle on bearing rolling bodies load distribution. Part 2: Simulation results. Acta Tech. Napoc. Ser. Appl. Math. Mech. 2013, 56, 795-800.

16. Xiaoli, R.; Jia, Z.; Ge, R. Calculation of radial load distribution on ball and roller bearings with positive, negative and zero clearance. Int. J. Mech. Sci. 2017, 131-132, 1-7. [CrossRef]

17. Fang, B.; Zhang, J.; Yan, K.; Wang, Z. A comprehensive study on the speed-varying stiffness of ball bearing under different load conditions. Mech. Mach. Theory 2019, 136, 1-13. [CrossRef]

18. Mitrović, R. Analysis of Influence of Elastic Deformations and Internal Radial Clearance of Deep Groove Ball Bearing on Load Distribution between Rolling Elements. Master's Thesis, Faculty of Mechanical Engineering, Belgrade, Serbia, 1987.

19. Mitrovic, R.; Ristivoevic, M.; Lazovic, T. Load distribution between rolling elements of deep groove ball bearings. Vestnik Mashinostroenya 2000, 3, 14-17.

20. Lazović, T.; Ristivojević, M.; Mitrović, R. Mathematical Model of Load Distribution in Rolling Bearing. FME Trans. 2008, 36, 189-196.

21. Ristivojević, M.; Mitrović, R. Load Distribution-Gear Pairs and Rolling Bearings; Faculty of Mechanical Engineering: Belgrade, Serbia, 2002.

22. Mitrović, R. Analysis of the influence of internal radial clearance on the static load rating of the rolling bearing. Facta Univ. Ser. Mech. Eng. 2001, 1, 1039-1047.

23. Zeljkovic, M.; Živkovic, A.; Borojev, L.J. Ball Bearings Static Behavior and Lifetime. Mach. Des. 2010, 2, 175-182. 
24. Tomovic, R. Research of the Impact of Rolling Bearing Construction Parameters on Their Correct Working Condition. Ph.D. Thesis, University of Niš, Niš, Serbia, 2009.

25. Tomović, R. Calculation of the boundary values of rolling bearing deflection in relation to the number of active rolling elements. Mech. Mach. Theory 2012, 47, 74-88. [CrossRef]

26. Tomović, R. Calculation of the necessary level of external radial load for inner ring support on q rolling elements in a radial bearing with internal radial clearance. Int. J. Mech. Sci. 2012, 60, 23-33. [CrossRef]

27. Tomović, R. Investigation of the Effect of Rolling Bearing Construction on Internal Load Distribution and the Number of Active Rolling Elements. Adv. Mater. Res. 2013, 633, 103-116. [CrossRef]

28. Tomović, R.; Miltenović, V.; Banić, M.; Miltenović, A. Vibration response of rigid rotor in unloaded rolling element bearing. Int. J. Mech. Sci. 2010, 52, 1176-1185. [CrossRef]

29. Tosić, M.; Tomović, R.; Jovanović, J. Static Analysis of Internal Load Distribution of the Single Row Deep Groove Ball Bearing. In Proceedings of the 3rd International Conference Mechanical Engineering in XXI Century, Niš, Serbia, 19-20 April 2015; pp. 173-178.

30. Tomović, R. A Simplified Mathematical Model for the Analysis of Varying Compliance Vibrations of a Rolling Bearing. Appl. Sci. 2020, 10, 670. [CrossRef]

31. Sheng, X.; Li, B.; Wu, Z.; Li, H. Calculation of ball bearing speed-varying stiffness. Mech. Mach. Theory 2014, 81, 166-180. [CrossRef]

32. Zhang, Z.M.; Hou, L.; Chen, Y.S. Bifurcations and hysteresis of varying compliance vibrations in the primary parametric resonance for a ball bearing. J. Sound Vib. 2015, 350, 171-184. [CrossRef]

33. ISO 5753-1. Rolling Bearings-Internal Clearance-Part 1: Radial Internal Clearance for Radial Bearings; International Organization for Standardization: Geneva, Switzerland, 2009.

(C) 2020 by the author. Licensee MDPI, Basel, Switzerland. This article is an open access article distributed under the terms and conditions of the Creative Commons Attribution (CC BY) license (http://creativecommons.org/licenses/by/4.0/). 